\title{
GENERALIZED LCM MATRICES
}

\author{
ANTAL BEGE
}

\begin{abstract}
Let $f$ be an arithmetical function. The matrix $[f[i, j]]_{n \times n}$ given by the value of $f$ in least common multiple of $[i, j], f([i, j])$ as its $i, j$ entry is called the least common multiple (LCM) matrix. We consider the generalization of this matrix where the elements are in the form $f(n,[i, j])$ and $f(n, i, j,[i, j])$.
\end{abstract}

\section{INTRODUCTION}

The classical Smith determinant was introduced in 1875 by H. J. S. Smith [12] who also proved that

(1)

$$
\operatorname{det}[(i, j)]_{n \times n}=\left|\begin{array}{cccc}
(1,1) & (1,2) & \cdots & (1, n) \\
(2,1) & (2,2) & \cdots & (2, n) \\
\cdots & \cdots & \cdots & \cdots \\
(n, 1) & (n, 2) & \cdots & (n, n)
\end{array}\right|=\varphi(1) \varphi(2) \cdots \varphi(n)
$$

where $(i, j)$ represents the greatest common divisor of $i$ and $j$, and $\varphi(n)$ denotes the Euler totient function.

The GCD matrix with respect to $f$ is

$$
[f(i, j)]_{n \times n}=\left[\begin{array}{cccc}
f((1,1)) & f((1,2)) & \cdots & f((1, n)) \\
f((2,1)) & f((2,2)) & \cdots & f((2, n)) \\
\cdots & \cdots & \cdots & \cdots \\
f((n, 1)) & f((n, 2)) & \cdots & f((n, n))
\end{array}\right] .
$$

There are quite a few generalized forms of GCD matrices, which can be found in several references [1, 3, 17, 8, 11].

H. J. S. Smith 12 also evaluated the determinant of

$$
[[i, j]]_{n \times n}=\left[\begin{array}{cccc}
{[1,1]} & {[1,2]} & \cdots & {[1, n]} \\
{[2,1]} & {[2,2]} & \cdots & {[2, n]} \\
\cdots & \cdots & \cdots & \cdots \\
{[n, 1]} & {[n, 2]} & \cdots & {[n, n]}
\end{array}\right]
$$

Date: August 24, 2011.

2010 Mathematics Subject Classification. Primary 11C20, 11A25, 15A36 Secondary $15 \mathrm{~A} 36$.

Key words and phrases. LCM matrix, Smith determinant, arithmetical function. 
and proved that

$$
\begin{aligned}
\operatorname{det}[[i, j]]_{n \times n} & =(n !)^{2} g(1) g(2) \cdots g(n)= \\
& =\prod_{k=1}^{N} \varphi(k) \prod_{p \mid k}(-p) .
\end{aligned}
$$

where $g(n)=\frac{1}{n} \sum_{d \mid n} d \mu(d), \mu(n)$ being the classical Möbius function. The structure of an LCM matrix $[[i, j]]_{n \times n}$ is the following (I. Korkee, P. Haukkanen [10])

$$
[[i, j]]_{n \times n}=\operatorname{diag}(1,2, \ldots, n) A A^{\top} \operatorname{diag}(12, \ldots, n),
$$

where $A=\left[a_{i j}\right]_{\mathfrak{n} \times \mathfrak{n}}$,

$$
a_{i j}=\left\{\begin{array}{ccc}
\sqrt{g(j)}, & \text { if } & j \mid i \\
0, & \text { if } j \chi_{i}
\end{array} .\right.
$$

The LCM matrix with respect to $f$ is

$$
[f[i, j]]_{n \times n}=\left[\begin{array}{cccc}
f([1,1]) & f([1,2]) & \cdots & f([1, n]) \\
f([2,1]) & f([2,2]) & \cdots & f([2, n]) \\
\cdots & \cdots & \cdots & \cdots \\
f([n, 1]) & f([n, 2]) & \cdots & f([n, n])
\end{array}\right]
$$

Results concerning LCM matrices appear in papers S. Beslin [2], K. Bourque, S. Ligh [4], W. Feng, S. Hong, J. Zhao [6] P. Haukkanen, J. Wang and J. Sillanpää [7].

In this paper we study matrices which have as variables the least common multiple and the indices

$$
[f(n,[i, j])]_{n \times n}=\left[\begin{array}{cccc}
f(n,[1,1]) & f(n,[1,2]) & \cdots & f(n,[1, n]) \\
f(n,[2,1]) & f(n,[2,2]) & \cdots & f(n,[2, n]) \\
\cdots & \cdots & \cdots & \cdots \\
f(n,[n, 1]) & f(n,[n, 2]) & \cdots & f(n,[n, n])
\end{array}\right]
$$

and the more general form matrices

$$
[f(n, i, j,[i, j])]_{n \times n}=\left[\begin{array}{cccc}
f(n, 1,1,[1,1]) & f(n, 1,2,[1,2]) & \cdots & f(n, 1, n,[1, n]) \\
f(n, 2,1,[2,1]) & f(n, 2,2,[2,2]) & \cdots & f(n, 2, n,[2, n]) \\
\ldots & \cdots & \cdots & \cdots \\
f(n, n, 1,[n, 1]) & f(n, n, 2,[n, 2]) & \cdots & f(n, n, n,[n, n])
\end{array}\right]
$$




\section{Generalized LCM matrices}

Theorem 2.1. For a given totally multiplicative aritmetical function $\mathrm{g}(\mathrm{n})$ let

Then

$$
f(n,[i, j])=g([i, j]) \sum_{k \leq \frac{n}{[i, j]}} g(k) .
$$

$$
[f(n,[i, j])]_{n \times n}=C_{n}^{\top} \operatorname{diag}(g(1), g(2), \ldots, g(n)) C_{n},
$$

where $C_{n}=\left[c_{i j}\right]_{n \times n}$

$$
c_{i j}=\left\{\begin{array}{ll}
1, & \text { if } j \mid i \\
0, & \text { if } j \times i
\end{array} .\right.
$$

For a determinant we have

$$
\operatorname{det}[f(n,[i, j])]_{n \times n}=g(1) g(2) \cdots g(n) .
$$

Proof. After multiplication, the general element of $A=\left(a_{i j}\right)_{\mathfrak{n} \times \mathfrak{n}}$,

$$
A=C_{n}^{\top} \operatorname{diag}(g(1), g(2), \ldots, g(n)) C_{n}
$$

is

$$
a_{i j}=\sum_{k=1}^{n} c_{k i} g(k) c_{k j}=\sum_{\substack{i|k \\ j| k \\ k \leq n}} g(k)=\sum_{\substack{[i, j] \mid k \\ k \leq n}} g(k)=\sum_{l \leq \frac{n}{i, j]}} g([i, j] l) .
$$

Because $\mathrm{g}(\mathrm{n})$ is totally multiplicative

$$
a_{i j}=g([i, j]) \sum_{\ell \leq \frac{n}{\lfloor i, j]}} g(\ell)=f([i, j]) .
$$

If we calculate the determinant of both parts of (21) we have (3).

\section{Particular cases}

Example 1. If $\mathrm{g}(\mathrm{n})=1$, then

$$
f(n,[i, j])=\left\lfloor\frac{n}{[i, j]}\right\rfloor,
$$

where $\lfloor x\rfloor$ denotes the integer part of $x$.

From Theorem 2.1 we have

$$
\begin{gathered}
{\left[\left\lfloor\frac{n}{[i, j]}\right\rfloor\right]_{n \times n}=C_{n}^{\top} \operatorname{diag}(1,1, \ldots, 1) C_{n},} \\
\operatorname{det}\left[\left\lfloor\frac{n}{[i, j]}\right\rfloor\right]_{n \times n}=1 .
\end{gathered}
$$


Example 2. If $\mathrm{g}(\mathrm{n})=\mathrm{n}$, then

$$
f(n,[i, j])=\frac{\left\lfloor\frac{n}{[i, j]}\right\rfloor\left\lfloor\frac{n}{[i, j]}+1\right\rfloor}{2} .
$$

The decomposition of generalized LCM matrix is

$$
[f(n,[i, j])]_{n \times n}=C_{n}^{T} \operatorname{diag}(1,2, \ldots, n) C_{n},
$$

and the determinant

$$
\operatorname{det}[f(n,[i, j])]_{n \times n}=n ! .
$$

Example 3. If $\mathrm{g}(\mathrm{n})=(-1)^{\Omega(\mathfrak{n})}$ is a Liouville function, then

$$
f(n,[i, j])=(-1)^{\Omega([i, j])} \sum_{k \leq \frac{n}{i j, j]}}(-1)^{\Omega(k)}
$$

and

$$
\begin{gathered}
{[\mathrm{f}(\mathrm{n},[i, j])]_{\mathrm{n} \times \mathfrak{n}}=C_{n}^{\top} \operatorname{diag}\left(1,-1, \ldots,(-1)^{\Omega(\mathrm{n})}\right) C_{n},} \\
\operatorname{det}[\mathrm{f}(\mathrm{n},[i, j])]_{\mathrm{n} \times \mathfrak{n}}=(-1)^{\sum_{k=1}^{n} \Omega(k)} .
\end{gathered}
$$

We remark that matrices related to the greatest integer function appeared in [9, 5].

Theorem 2.2. For a given totally multiplicative function $\mathrm{g}$ let

$$
f(n, i, j,[i, j])=\sum_{k \leq n} g(k)-g(i) \sum_{l \leq \frac{n}{i}} g(l)-g(j) \sum_{l \leq \frac{n}{j}} g(l)+g([i, j]) \sum_{k \leq \frac{n}{i, j]}} g(k) .
$$

Then

$$
[f(n, i, j,[i, j])]_{n \times n}=D_{n}^{\top} \operatorname{diag}[g(1), g(2), \ldots, g(n)] D_{n},
$$

where $D_{\mathfrak{n}}=\left[d_{i j}\right]_{\mathfrak{n} \times \mathfrak{n}}$,

$$
d_{i j}=\left\{\begin{array}{ll}
1, & \text { if } j \times i \\
0, & \text { if } j \mid i
\end{array} .\right.
$$

Proof. After multiplication the general element of the matrix

$$
A=\left[a_{i j}\right]_{n \times n}=D_{n}^{\top} \operatorname{diag}[g(1), g(2), \ldots, g(n)] D_{n}
$$


is

$$
\begin{aligned}
a_{i j}= & \sum_{\substack{i \nmid k \\
j \nmid k \\
k \leq n}} g(k)=\sum_{k \leq n} g(k)-\sum_{i \mid k} g(k)-\sum_{j \mid k} g(k)+\sum_{\substack{i|k \\
j| k \\
k \leq n \\
k \leq n}} g(k)= \\
= & \sum_{k \leq n} g(k)-\sum_{i l \leq n} g(i l)-\sum_{j l \leq n} g(j l)+\sum_{\substack{[i, j] \mid k \\
k \leq n}} g(k)
\end{aligned}
$$

The total multiplicativity of $g$ implies,

$$
\begin{aligned}
a_{i j} & =\sum_{k \leq n} g(k)-g(i) \sum_{l \leq \frac{n}{i}} g(l)-g(j) \sum_{l \leq \frac{n}{j}} g(l)+g([i, j]) \sum_{k \leq \frac{n}{[i, j]}} g(k)= \\
& =f(n, i, j,[i, j]) .
\end{aligned}
$$

\section{Particular cases}

Example 4. If $\mathrm{g}(\mathrm{n})=1$, then

$$
f(n, i, j,[i, j])=\tau(n)-\tau\left(\left\lfloor\frac{n}{i}\right\rfloor\right)-\tau\left(\left\lfloor\frac{n}{j}\right\rfloor\right)+\left\lfloor\frac{n}{[i, j]}\right\rfloor,
$$

where $\tau(n)=\sum_{d \mid n} 1$. By Theorem 2.2

$$
\left[f\left(n, i, j,\left\lfloor\frac{n}{[i, j]}\right\rfloor\right)\right]_{n \times n}=D_{n}^{\top} \operatorname{diag}(1,1, \ldots, 1) D_{n} .
$$

Example 5. If $\mathrm{g}(\mathrm{n})=\mathrm{n}$, then

$$
f(n, i, j,[i, j])=\sigma(n)-\sigma\left(\left\lfloor\frac{n}{i}\right\rfloor\right)-\sigma\left(\left\lfloor\frac{n}{j}\right\rfloor\right)+\frac{\left\lfloor\frac{n}{[i, j]}\right\rfloor\left\lfloor\frac{n}{[i, j]}+1\right\rfloor}{2},
$$

where $\sigma(n)=\sum_{d \mid n} d$.

The general form of a generalized LCM matrix is

$$
[f(n, i, j,[i, j])]_{n \times n}=D_{n}^{\top} \operatorname{diag}(1,2, \ldots, n) D_{n} .
$$


Example 6. If $\mathrm{g}(\mathrm{n})=(-1)^{\Omega(\mathrm{n})}$ is the Liouville function then

$$
\begin{aligned}
f(n, i, j,[i, j]) & =\sum_{k \leq n}(-1)^{\Omega(k)}-(-1)^{\Omega(i)} \sum_{l \leq \frac{n}{i}}(-1)^{\Omega(l)}-(-1)^{\Omega(j)} \sum_{l \leq \frac{n}{j}}(-1)^{\Omega(l)} g+ \\
& +(-1)^{\Omega([i, j])} \sum_{k \leq \frac{n}{[i, j]}}(-1)^{\Omega(k)}
\end{aligned}
$$

and

$$
\left[f(n, i, j,[i, j]]_{n \times n}=D_{n}^{\top} \operatorname{diag}\left(1,-1, \ldots,(-1)^{\Omega(n)}\right) D_{n} .\right.
$$

Remark 2.1. Due to the fact that the first line of the matrix $[f(n, i, j,[i, j])]_{n \times n}$ contains only 0 -s, the determinant of the matrix will always be 0 .

Acknowledgement. This research was supported by the grant of Sapientia Foundation, Institute of Scientific Research.

\section{REFERENCES}

[1] A. Bege, Generalized GCD matrices Acta Univ. Sapientiae, Math., 2 2010, 160-167.

[2] S. Beslin, Reciprocal GCD matrices and LCM matrices, Fibonacci Quart., 29 1991, 271-274.

[3] K. Bourque, S. Ligh, Matrices associated with classes of arithmetical functions, J. Number Theory, 45 1993, 367-376.

[4] K. Bourque, S. Ligh, Matrices associated with multiplicative functions, Linear Algebra Appl., 216 1995, 267-275.

[5] L. Carlitz, Some matrices related to the greatest integer function, J. Elisha Mitchell Sci. Soc., 76 1960, 5-7.

[6] W. Feng, S. Hong, J. Zhao, Divisibility properties of power LCM matrices by power GCD matrices on gcd-closed sets, Discrete Math., 309 2009, 2627-2639.

[7] P. Haukkanen, J. Wang and J. Sillanpää, On Smiths determinant Linear Algebra Appl., 258 1997, 251-269.

[8] S. Hong, Factorization of matrices associated with classes of arithmetical functions, Colloq. Math., 98 2003, 113-123.

[9] E. Jacobsthal, Über die grösste ganze Zahl. II. (German), Norske Vid. Selsk. Forh., Trondheim, 30 1957, 6-13.

[10] I. Korkee, P. Haukkanen, On meet and join matrices associated with incidence functions, Linear Algebra Appl., 372 2003, 127-153.

[11] J. S. Ovall, An analysis of GCD and LCM matrices via the $L D L^{T}$-factorization, Electron. J. Linear Algebra, 11 2004, 51-58.

[12] H. J. S. Smith, On the value of a certain arithmetical determinant, Proc. London Math. Soc., 7 1875/76, 208-212.

Department of Mathematics and Informatics, University of SapienTia, P.O. 9, P. O. Box 4, RO-540485 Tîrgu Mureş, Romania

E-mail address: abege@ms.sapientia.ro 